\title{
Phenomenology of a Composite Higgs Model
}

\section{Luigi Del Debbio*}

Higgs Centre for Theoretical Physics

The University of Edinburgh

Edinburgh EH9 3FD, Scotland, UK

E-mail: luigi.del.debbioded.ac.uk

\section{Christoph Englert}

School of Physics and Astronomy

The University of Glasgow

Glasgow, G12 8QQ, Scotland, UK

E-mail: christoph.englerteglasgow.ac.uk

\section{Roman Zwicky}

Higgs Centre for Theoretical Physics

The University of Edinburgh

Edinburgh EH9 3FD, Scotland, UK

E-mail: roman.zwicky@ed.ac.uk

Several UV complete models of physics beyond the Standard Model are currently under scrutiny, their low-energy dynamics being compared with the experimental data from the LHC. Lattice simulations can play a role in these studies by providing a first principles computations of the low-energy constants that describe this low-energy dynamics. In this work, we study in detail a specific model recently proposed by Ferretti [1], and discuss the potential impact of lattice calculations.

34th annual International Symposium on Lattice Field Theory

24-30 July 2016

University of Southampton, UK

\footnotetext{
*Speaker.
} 


\section{Introduction}

Strongly-interacting dynamics is one of the possible scenarios describing the breaking of the electro-weak (EW) symmetry observed in the Standard Model (SM): new physics beyond the Standard Model (BSM) is the manifestation of a new theory in a nonperturbative regime, giving rise to models known as composite Higgs models. The dynamics of the low-lying states in the spectrum of the strongly-interacting theory is described by effective field theories (EFT), where the nonperturbative dynamics is encoded in a number of low-energy constants (LECs). EFTs are the preferred tool to compare strongly-interacting models with experimental data. Measurements translate directly into constraints on the LECs, without ever having to specify the details of the underlying theory, which is often referred to as the UV completion. Traditionally, UV completions are supposed to be gauge theories coupled to matter in one, or several, representations of the gauge group. We shall refer to the charge under this new gauge group as hypercolor, while we will use the word color for the usual QCD sector of the SM.

On the other hand, given a UV completion in the form of a hypercolor theory, first-principle information on the spectrum, and the LECs of the EFT, can be determined from lattice Monte Carlo simulations of the UV completion. These simulations are computationally expensive, and it is desirable to determine the relevant observables, and their desired precision, from phenomenological studies.

In this work we address the question of the potential impact of lattice simulations within the particular model presented by Ferretti in Ref. [1]. We write down the EFT that encodes the pattern of spontaneous symmetry breaking in the UV completion, and analyse the bounds on the LECs from current LHC data. While similar studies have already been performed, our analysis focusses especially on finding the experimental bounds on the quantities that can be computed in lattice simulations.

\section{Low-energy spectrum}

Let us briefly recall the low-energy description of the model in Ref. [1]. The effective action includes the nonlinear sigma model action describing the Nambu-Goldstone bosons (NGB) of the strong theory, and their self-interactions, and terms that describe their coupling to the SM particles. The UV completion is an SU(4) gauge theory, with 5 Weyl fermions $\left(\psi_{m n}^{I}\right)$ in the twoindex antisymmetric representation of the hypercolor group, and 3 Dirac fermions (represented as a pair of Weyl fermions $\chi_{m}^{a}, \bar{\chi}_{m}^{a^{\prime}}$ ) in the fundamental representation of the hypercolor group. In the expressions above, the flavor indices are $I=1, \ldots, 5, a=1, \ldots, 3$, and $a^{\prime}=1, \ldots, 3$; the indices $m, n=1, \ldots, 4$ are hypercolor indices. The theory is symmetric under global transformations belonging to

$$
G_{F}=\mathrm{SU}(5) \times \mathrm{SU}(3) \times \mathrm{SU}(3)^{\prime} \times \mathrm{U}(1)_{X} \times \mathrm{U}(1)^{\prime} .
$$

The charges of the matter fields under the various symmetry transformations are explained in detail in Ref. [1]. The expected spontaneous symmetry breaking pattern of the model takes the form

$$
G_{F} / H_{F}=\left(\frac{\mathrm{SU}(5)}{\mathrm{SO}(5)}\right) \times\left(\frac{\mathrm{SU}(3) \times \mathrm{SU}(3)^{\prime}}{\mathrm{SU}(3)_{c}}\right) \times\left(\frac{\mathrm{U}(1)_{X} \times \mathrm{U}(1)^{\prime}}{\mathrm{U}(1)_{X}}\right)
$$


induced by the condensates $\left\langle\varepsilon^{m n p q} \psi_{m n}^{I} \psi_{p q}^{J}\right\rangle \propto \delta^{I J}$, and $\left\langle\bar{\chi}_{m}^{a^{\prime}} \chi_{m}^{a}\right\rangle \propto \delta^{a^{\prime} a}$.

The unbroken subgroup $H_{F}=\mathrm{SO}(5) \times \mathrm{SU}(3)_{c} \times \mathrm{U}(1)_{X}$ must contain the SM group. The EW gauge group $\mathrm{SU}(2)_{L} \times \mathrm{U}(1)_{Y}$ is embedded in the unbroken $\mathrm{SO}(5)$ by considering the subgroup $\mathrm{SO}(4) \simeq \mathrm{SU}(2)_{L} \times \mathrm{SU}(2)_{R}$, and then identifying a $\mathrm{U}(1)$ subgroup generated by $T_{R}^{3}$, the third generator of $\mathrm{SU}(2)_{R}$, and setting $Y=T_{R}^{3}+X$. The unbroken vector subgroup $\mathrm{SU}(3)_{c}$ is gauged, and identified with the QCD subgroup of the SM.

The $14 \mathrm{NGB}$ in the coset $\mathrm{SU}(5) / \mathrm{SO}(5)$ can be classified according to their $\mathrm{SM} \mathrm{SU}(2)_{L} \times$ $\mathrm{U}(1)_{R}$ charges:

$$
\mathbf{1 4} \rightarrow \mathbf{1}_{0}+\mathbf{2}_{ \pm 1 / 2}+\mathbf{3}_{0}+\mathbf{3}_{ \pm 1}=\left(\eta, H, \Phi_{0}, \Phi_{ \pm}\right) .
$$

In composite models the Higgs boson is the NGB denoted by $H$ in the list above. The field $H$ is a doublet under $\mathrm{SU}(2)_{L}$, and can be written as a two-component complex field $H=\left(H_{+}, H_{0}\right)$. If we consider the hypercolor theory with massless fermions in isolation, the NGB are exactly massless. The potential for the $H$ field, and more generally the mass of all NGBs, is generated by the coupling of the hypercolor theory to the SM fields. Note that the $\mathrm{NGB}$ in the $\mathrm{SU}(3) \times \mathrm{SU}(3)^{\prime} / \mathrm{SU}(3)_{c}$ coset are color charged massless states that characterize this particular hypercolor theory. We will briefly discuss their properties below.

The spin 1/2, color triplet states of the hypercolor theory are natural candidates to play the role of top partners, and hence generate the mass of the heaviest quark. It is often assumed, when building EFTs, that there exists one such state of mass $M$, which is lighter than the typical scale of the hypercolor theory, $\Lambda_{\mathrm{HC}}$. A lattice study of the spectrum of the hypercolor theory would yield unambiguous predictions about the spectrum of expected resonances. In the context of an EFT description of the low-energy dynamics, the top partner is introduced in the effective action as a Dirac fermion field $\Psi$ transforming in the $(\mathbf{5 , 3})_{2 / 3}$ representation of $H_{F}$. The SM quantum numbers of this field can be found by decomposing the $(\mathbf{5}, \mathbf{3})_{2 / 3}$ representation into irreducible representations of $G_{\mathrm{SM}} \subset H_{F}$ :

$$
(\mathbf{5}, \mathbf{3})_{2 / 3} \rightarrow(\mathbf{3}, \mathbf{2})_{7 / 6}+(\mathbf{3}, \mathbf{2})_{1 / 6}+(\mathbf{3}, \mathbf{1})_{2 / 3}
$$

where the numbers on the RHS denote the irreducible representations of $\mathrm{SU}(3)_{c} \times \mathrm{SU}(2)_{L} \times \mathrm{U}(1)_{Y}$.

As usual the NGB are combined in a field $\Sigma=\exp \left(\frac{i \Pi}{f}\right)$, where $f$ is the NGB decay constant of the hypercolor theory, and hence one of the LEC in the effective action. $\Sigma$ transforms non-linearly under global SU(5) transformations. It is convenient to write the effective action in terms of the field $U=\Sigma \Sigma^{T}$, which transforms according to $U \mapsto g U g^{T}$ for $g \in \mathrm{SU}(5)$. The coupling to the SM gauge bosons is obtained by promoting the ordinary derivatives in the usual chiral lagrangian to covariant derivatives:

$$
\mathscr{L} \supset \frac{f^{2}}{16} \operatorname{tr}\left[\left(D_{\mu} U\right)^{\dagger} D^{\mu} U\right]
$$

where

$$
D_{\mu} U=\partial_{\mu} U-i g W_{\mu}^{a}\left[T_{L}^{a}, U\right]-i g^{\prime} B_{\mu}\left[T_{R}^{3}, U\right] .
$$

The mass term for the fermions and the coupling to the SM fermions is

$$
\mathscr{L} \supset \frac{M}{2} \bar{\Psi} \Psi+\lambda_{q} f \overline{\hat{q}}_{L} \Sigma \Psi_{R}+\lambda_{t} f \overline{\hat{t}}_{R} \Sigma^{*} \Psi_{L},
$$


where $\hat{q}_{L}$ and $\hat{t}_{R}$ are spurionic embedding of the SM quarks in the $\mathbf{5}$ and $\overline{\mathbf{5}}$ representations of SU(5) respectively. The mass of the hypercolor state $M$ is another LEC that can be determined from lattice studies of the spectrum of the theory. In this preliminary study, we will simply scan over a sensible range for $M$. Likewise $\lambda_{q}$ and $\lambda_{t}$ are LECs that determine the mass of the top quark.

The contributions of the SM particles to the Coleman-Weinberg potential of the NGBs are responsible for the misalignment of the vacuum, which leads to EW symmetry breaking. In particular only the fermionic couplings are responsible for negative contributions to the potential, which are necessary to generate a non-vanishing vev for the $H_{0}$ component. Following the notation in Ref. [1], we set $H_{0}=h / \sqrt{2}$, and all other fields to zero. The couplings of the field $h$ to the SM gauge bosons, and fermions are as follows:

$$
\begin{aligned}
\operatorname{tr}\left[U(h) W_{\mu} U(h)^{\dagger} W_{\mu}\right] & =\frac{1}{2}[1+\cos (2 h / f)] W_{\mu}^{c} W_{\mu}^{c}, \\
\overline{\hat{q}}_{L} U(h) \hat{t}_{R}+\overline{\hat{t}}_{R} U(h)^{*} \hat{q}_{L} & =\frac{1}{\sqrt{2}} \sin (2 h / f)\left(\bar{t}_{L} t_{R}+\bar{t}_{R} t_{L}\right) .
\end{aligned}
$$

The Coleman-Weinberg potential is parametrized by two LECs, $\alpha$ and $\beta$ :

$$
V(h) \propto \alpha \cos (2 h / f)-\beta \sin ^{2}(2 h / f) .
$$

The LECs encode the contributions of the SM sector to the potential, in a way that is analogous to the electromagnetic corrections to the pion mass [2]. They can be computed from field correlators as described in Ref. [3]:

$$
\begin{aligned}
2 \alpha & =-C_{L R}\left(3 g^{2}+g^{\prime 2}\right)<0, \\
\beta & =-2 y^{2} C_{\mathrm{top}} .
\end{aligned}
$$

Clearly EW symmetry breaking can only occur if $\alpha+2 \beta>0$. Moreover the value of the Higgs vev in units of $f$ is determined by these constants. The LECs in the equations above can be computed from first principles from correlators in the UV complete theory. For instance

$$
C_{L R}=\frac{3}{(4 \pi)^{2}} \int_{0}^{\infty} d q^{2} q^{2} \Pi_{L R}\left(q^{2}\right)+\mathscr{O}\left(g^{4}, g^{4}\right),
$$

where $\Pi_{L R}$ is defined from the Lorentz decomposition of the current/current correlators:

$$
\left(q^{2} \delta_{\mu v}-q_{\mu} q_{v}\right) \Pi_{L R}\left(q^{2}\right)=\int d^{D} x e^{i k x} \operatorname{tr}\left\langle J_{\mu}^{R}(x) J_{v}^{L}(0)\right\rangle,
$$

and $J_{\mu}^{R, L}(x)=\bar{\psi} \gamma_{\mu} \frac{1 \pm \gamma_{5}}{2} \psi(x)$. Similarly, $C_{\text {top }}$ can be computed on the lattice, and therefore the issue of EW dynamical symmetry breaking can be resolved by numerical simulations.

Finally note that diagonalising the mass term in the effective action yields the top mass, at leading order in $v$,

$$
m_{t}=\frac{\sqrt{2} M f \lambda_{q} \lambda_{t}}{\sqrt{M^{2}+\lambda_{q}^{2} f^{2}} \sqrt{M^{2}+\lambda_{t}^{2} f^{2}}} v
$$

or, equivalently,

$$
m_{t} / v=\frac{\sqrt{2} \rho_{M} \lambda_{q} \lambda_{t}}{\sqrt{1+\lambda_{q}^{2} \rho_{M}^{2}} \sqrt{1+\lambda_{t}^{2} \rho_{M}^{2}}}
$$


where we have introduced the ratio $\rho_{M}=f / M$, which can be extracted from numerical simulations.

Introducing spurions $\hat{q}_{L}^{i} \in \mathbf{2 4}, \hat{u}_{R}^{i} \in \mathbf{1 0}$, and $\hat{d}_{R}^{i} \in \overline{\mathbf{1 0}}$, where $i=1,2,3$ denotes the quark family, a mass term for the bottom quark can be introduced

$$
\mathscr{L} \supset \sqrt{2} \mu_{b} \operatorname{tr}\left(\overline{\hat{q}}_{L}^{3} U \hat{d}_{R}^{3}+\text { h.c. }\right) ;
$$

the new LEC, $\mu_{b}$, is determined by requiring that the correct value is recovered for the bottom quark mass.

\section{Constraints from data}

Expanding the exponential in $U$, the effective lagrangian for the Higgs field reduces to the socalled minimal composite Higgs model (MCHM) [4, 5, 6]. The Higgs coupling to the EW gauge bosons is rescaled by a factor $\sqrt{1-\xi}$ compared to the SM values, where $\xi=v^{2} / f^{2}$. Bounds on $\xi$ from current experimental data have been studied in detail in the context of MCHM, see e.g. [7].

As discussed in the previous section, this specific model predicts additional PNGBs, i.e. additional charged Higgs particles, whose masses are set by the EW contributions to the ColemanWeinberg potential. The color octet of hyper-pions complete the low-energy spectrum of the theory, the lower bound on their mass being currently in the multi-TeV regime [8, 9].

Assuming e.g. a mass $m \approx 200 \mathrm{GeV}$ for the exotic Higgses, and taking into account the experimental constraints on $\xi$, the production cross sections for these particles can be computed. Even for the lightest values of $m$, we find production cross sections of the order of $100 \mathrm{fb}$, which decrease exponentially for higher masses. This is reassuringly consistent with the fact that these states have not been observed experimentally.

The customary observable for studies of BSM phenomenology is the signal strength in a given channel, which is defined as the ratio of the expected events in the BSM extended model to the events predicted by the SM. The additional Higgs particles discussed above modify the $h \rightarrow W W, \gamma \gamma, Z Z$ signal strengths. For this preliminary report we focus on these three channels only, as examples of possible applications. In each channel we compute the signal strength predicted by the effective theory above. The results of our study are shown in Figs. 1, 2 and 3. In all plots the LHC data are displayed by the yellow band as a function of $\xi$, or, equivalently, $f$. The blue band is obtained by scanning over the values of the LECs. More precisely, we vary $M$ in the range $[1.5,3.5] \mathrm{TeV}, \lambda_{t}$ in the range $[0,4 \pi]$. The value of $\lambda_{q}$ is then fixed by the mass of the top quark, as shown in Eq. 2.16. Finally $\mu_{b}$ is engineered to reproduce the correct bottom quark mass of $4.7 \mathrm{GeV}$.

The plots show a bit of tension between the signal strength meaurements by ATLAS and CMS and the prediction in the model. In particular, the relatively small signal strength in the $W W$ and $Z Z$ channels is a problem that all MCHM-type scenarios face. However, the presence of the newly charged scalar states introduces additional freedom to obtain a signal strength in the diphoton channel that is comparable to the observed limits. The width of the blue band highlights the sensitivity of the Higgs branching to photon pairs. On the other hand, since $h \rightarrow \gamma \gamma$ is only a small partial decay width the impact on the prompt $h \rightarrow Z Z, W W$ decays is only small. Hence, the size of the blue band (signalling the scan's spread) in these channels is a lot more narrow. 


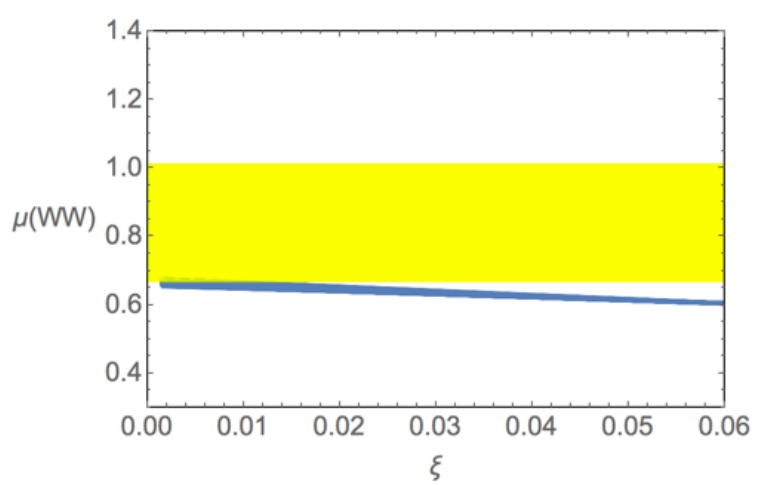

Figure 1: Signal strength $\mu(W W)$ for the decay into $h \rightarrow W W$ gauge bosons. The yellow band is the error in the LHC data, while the blue band is the error due to the scan in the parameters of the EFT.

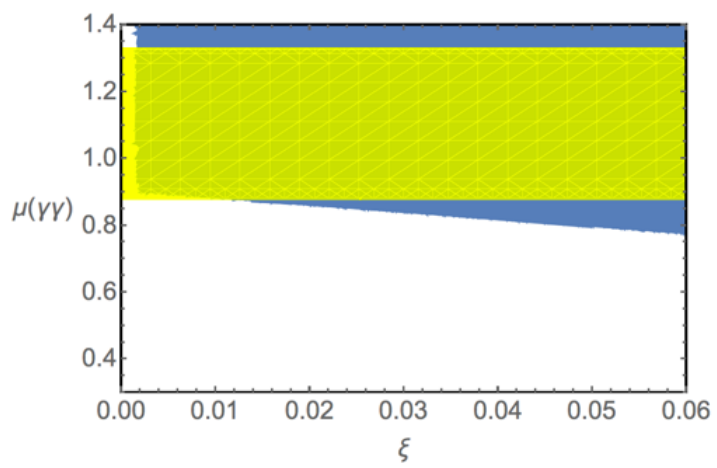

Figure 2: Signal strength $\mu(\gamma \gamma)$ for the decay into $h \rightarrow \gamma \gamma$ gauge bosons. The yellow band is the error in the LHC data, while the blue band is the error due to the scan in the parameters of the EFT.

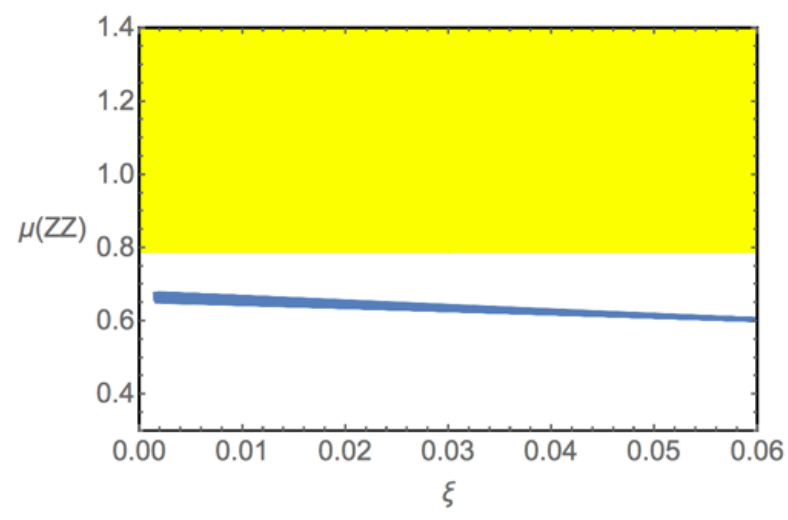

Figure 3: Signal strength $\mu(Z Z)$ for the decay into $h \rightarrow Z Z$ gauge bosons. The yellow band is the error in the LHC data, while the blue band is the error due to the scan in the parameters of the EFT. 


\section{Conclusions}

The preliminary results of our study suggest that the UV theory considered here is compatible with the data analysed so far by the experiments at the LHC, for a sensible range of the couplings in the effective action, despite showing some tension e.g. in the $h \rightarrow Z Z$ channel, and the $h \rightarrow W W$ to a lesser extent. Currently, the main source of uncertainty is the experimental error in the data, while the variations in the theoretical predictions due to the scan in the space of parameters seems to be rather smaller.

We have identified a number of LECs that appear in the effective action, and have discussed the possibility of measuring them in lattice simulations of the UV complete underlying theory. Some of the LEC like the condensates, $C_{L R}, C_{\text {top }}$ allow us to check the pattern of symmetry breaking, and the generation of the correct Higgs potential. Note that a robust prediction of the sign of the combination $\alpha+2 \beta$ would already yield valuable information. Lattice results would also constrain the region that needs to be explored when scanning over the space of LECs. In particular a determination of $\xi$ and $\rho_{M}$ would reduce the scan to a lower-dimensional subspace, and therefore could constrain the model substantially.

As the experimental errors decrease, lattice simulations can lead to stringent tests of BSM models with a UV completion.

\section{References}

[1] G. Ferretti, JHEP 1406 (2014) 142 doi:10.1007/JHEP06(2014)142 [arXiv:1404.7137 [hep-ph]].

[2] T. Das, G. S. Guralnik, V. S. Mathur, F. E. Low and J. E. Young, Phys. Rev. Lett. 18 (1967) 759. doi:10.1103/PhysRevLett.18.759

[3] M. Golterman and Y. Shamir, Phys. Rev. D 91 (2015) no.9, 094506 doi:10.1103/PhysRevD.91.094506 [arXiv:1502.00390 [hep-ph]].

[4] R. Contino, Y. Nomura and A. Pomarol, Nucl. Phys. B 671 (2003) 148 doi:10.1016/j.nuclphysb.2003.08.027 [hep-ph/0306259].

[5] K. Agashe, R. Contino and A. Pomarol, Nucl. Phys. B 719 (2005) 165 doi:10.1016/j.nuclphysb.2005.04.035 [hep-ph/0412089].

[6] R. Contino, L. Da Rold and A. Pomarol, Phys. Rev. D 75 (2007) 055014 doi:10.1103/PhysRevD.75.055014 [hep-ph/0612048].

[7] G. Aad et al. [ATLAS Collaboration], JHEP 1511 (2015) 206 doi:10.1007/JHEP11(2015)206 [arXiv:1509.00672 [hep-ex]].

[8] V. Khachatryan et al. [CMS Collaboration], Phys. Lett. B 747 (2015) 98 doi:10.1016/j.physletb.2015.04.045 [arXiv:1412.7706 [hep-ex]].

[9] The ATLAS collaboration [ATLAS Collaboration], ATLAS-CONF-2016-084. 